山्山FFRANÇAISE

$\supset \mathrm{DE}$

$\simeq$ PÉDAGOGIE

\section{Revue française de pédagogie}

Recherches en éducation

154 | janvier-mars 2006

La construction des politiques d'éducation : de nouveaux rapports entre science et politique

\title{
Entre recherche, pratique et politique : le Conseil supérieur de l'éducation du Québec, une démarche originale
}

Between research, practice and politics: the Quebec's Council of Education, an original approach

Zwischen Forschung, Praxis und Politik: das Obererziehungsamt in Quebec, eine originale Vorgehensweise

Entre investigación, práctica y política : el Consejo superior de la educación de Quebec, un proceso original

\section{Céline Saint-Pierre}

\section{OpenEdition \\ Journals}

Édition électronique

URL : http://journals.openedition.org/rfp/132

DOI : $10.4000 /$ rfp. 132

ISSN : 2105-2913

Éditeur

ENS Éditions

Édition imprimée

Date de publication : 1 mars 2006

Pagination : 73-84

ISBN : 2-7342-1039

ISSN : 0556-7807

\section{Référence électronique}

Céline Saint-Pierre, «Entre recherche, pratique et politique : le Conseil supérieur de l'éducation du Québec, une démarche originale », Revue française de pédagogie [En ligne], 154 I janvier-mars 2006, mis en ligne le 01 mars 2010, consulté le 19 avril 2019. URL : http://journals.openedition.org/rfp/132 ; DOI : $10.4000 / r f p .132$ 


\title{
Entre recherche, pratique et politique : le Conseil Supérieur de I'éducation du Q Uébec, Une démarche originale
}

\author{
Céline Saint-Pierre
}

Dans cet article, nous faisons état d'un processus original et unique de co-construction des savoirs en éduc aion. Nous tentons de Cerner le jeu des rapports entre la recherche comme dém arche de production de savoirs savants, les interventions des acteurs du système d'éduc aion, porteurs de savoirs pratiques et l'élaboration de politiques publiques au sein de l'appareil gouvernemental. Pour ce faire, nous analysons le mode de travail propre au Conseil supérieur de l'éducation, un conseil aviseur au ministre de l'Éducation du gouvernement du Québec. Nous présentons des exemples qui permettent d'expliciter le jeu des rapports entre ces trois éléments et nous concluons par quelques pistes susceptibles de les consolider tout en montrant les difficultés d'évaluer son pouvoir d'influence auprès des décideurs politiques et de la société civile.

De Scripte urs (TEE) : autorité Compétente, développement de l'éducation, production sociale du savoir, Québec, réforme.

\section{INTRODUCTION}

Nous nous proposons de faire état d'un processus unique et original de co-Construction des savoirs dont le résultat vise à soutenir l'élaboration de politiques publiques en éduc aion. Nous tenterons de Cerner le jeu des rapports entre la recherche Comme démarche de production de savoirs savants, les interventions des acteurs du système d'éduc aion porteurs de savoirs pratiques et l'élaboration de politiques publiques. À Cette fin, nous nous intéresserons plus particulièrement à un organisme assez unique, soit le Conseil supérieur de l'éducation, un Conseil aviseur auprès du ministre de l'Éducation et du gouvernement du Québec. Cet organisme est né en 1964 à la suite d'une recomm andation du rapport de la Commission royale d'enquête sur l'enseignement dans la province de Québec. II faut dire ici que peu de gouvernements se sont dotés d'un tel type d'organisme dans leur structure et l'intérêt qu'il susCite dans d'autres pays et auprès de nombreux observateurs relève à la fois de la nature de sa mis sion et de l'originalité de son fonctionnement. En plus d'être un Conseiller de l'État, le législateur, dans sa décision de mettre sur pied Cet organisme (SaintPierre, 2004), lui a attribué le rôle de favoriser la participation démocratique de la société civile à la définition des politiques en éducation. La réalisation de 
Ce volet de sa mission repose en grande partie sur sa c a acité à mettre en œuvre un véritable proces sus de Co-Construction et de CirCulation des savoirs dont les enjeux prennent place dans le champ éduc af Considéré Comme espace socio-politique. Le rapport de Cette Commission, mieux Connu sous le nom de rapport Parent, a appuyé sa recommandation sur sa Conviction qu'il fallait assurer, au-delà du $m$ inistère de l'Éducation, une "liaison entre le grand public et le gouvernement, l'opinion publique ayant, en régime démocratique, le droit et le devoir de s'exprimer sur une question aussi vitale que l'éducation." (Québec, 1993) Cet objectif allait devoir s'incarner dans une mission et un mode de fonctionnement favorisant l'interaction entre la recherche, les pratiques des acteurs de l'éducation et l'élaboration des politiques publiques en éducation.

Dans un premier temps, nous examinerons quel mode de travail a été $m$ is en place par cet organisme pour réaliser sa mission. Nous verrons que la forme de sa structure et la Composition de ses instances font se Côtoyer Chercheurs et praticiens de l'éducation dans la production des avis et des rapports destinés au ministre de l'Éducation. La réalisation de la $m$ ission Centrale du Conseil supérieur de l'éducation repose en très grande partie sur la qualité expérientielle, les Compétences intellectuelles et la disponibilité des membres de ses diverses instances, issus en très grande majorité $d u$ milieu même de l'éducation. Ils sont appelés à contribuer à l'élaboration de diagnostics et d'évaluations de situations à partir d'un examen Critique des pratiques de leur propre milieu; par ailleurs, il leur est demandé d'appuyer leur analyse sur les résultats des recherches existantes sur le sujet traité ou réalisées par l'équipe de recherche du Conseil. Les Connaissances ainsi Co-produites par les Chercheurs et les praticiens Constituent le $m$ atériau premier et essentiel au travail des membres du Conseil et de ses Commissions Chargés de formuler des orientations pour l'action sous forme de recomm andations. Celles-Ci sont déposées en premier lieu au ministre de l'Éducation du Québec et diffusées par la suite aux acteurs de l'ensemble du système d'éducation. Tous les avis et rapports sont rendus publics et font l'objet d'une large diffusion.

Dans un deuxième temps, nous présenterons quelques exemples concrets qui permettront d'expliCiter Comment le Conseil produit ses avis et de dégager, dans la mesure du possible, l'influence qu'il a exercé sur l'élaboration de Certaines politiques éducatives. Nous y verrons comment la recherche joue un rôle-clé dans la validation des recommandations du Conseil et permet aussi d'ouvrir de nouveaux champs de Connaissance qui orienteront les Contenus des politiques éducatives.

Enfin, nous terminerons par la formulation de quelques pistes visant la consolidation des rapports entre la recherche, les milieux de pratique et l'élaboration des politiques éducatives. L'une d'elles mise sur l'acquisition de compétences spécifiques par les acteurs de terrain, une composante du profes. sionnalisme collectif. Une autre concerne le statut des sciences de l'éducation dans la production des savoirs et la place que les facultés d'éducation occupent au sein de l'institution universitaire. Et enfin, nous concluons en montrant que, si pour le Conseil, l'évaluation de sa production au regard de la réalisation de sa mission est une garantie néces. saire à l'exercice de l'influence recherchée, le choix d'indicateurs pour ce faire n'est pas simple.

\section{UNE DÉM ARCHE ORIGINALE DE PRODUCTION DES CONNAISSANCES : LE MODE DE TRAV AIL DU CONSEIL SUPÉRIEUR DE L'ÉDUCATION}

Pour bien saisir l'originalité du Conseil supérieur de l'éducation, il faut en exposer la mission et le fonctionnement, puisqu'il n'existe pas d'organisme identique dans d'autres pays. Il s'agit d'un organisme consultatif dont le rôle est de préparer et de soumettre, à son initiative, des avis au ministre de l'Éducation. Ces avis portent sur toutes questions relatives à l'éducation à tous les ordres d'enseignement et à l'éducation des adultes, ainsi qu'à l'éducation à l'extérieur du système formel. Par ailleurs, la loi Constitutive du Conseil supérieur de l'éducation oblige aussi le ministre à consulter le Conseil sur un Certain nombre de questions, telle que les projets de règlements pédagogiques et leur modification. La loi prévoit que l'État et le ministre de l'Éducation se doivent de respecter l'indépendance et l'autonomie Complète de l'organisme et d'observer la non-ingé. rence politique dans ses travaux.

De par la Composition de ses instances, le législateur a voulu en faire un organisme de participation démocratique, ce qui le situe à l'opposé d'un bureau d'experts scientifiques ou de spécialistes. La Centaine de membres qui les Composent sont pour la plupart des acteurs de première ligne $d u$ milieu de l'éducation et une minorité provient de divers autres secteurs de la société québécoise. Ils sont enseignants et directeurs d'établissements à tous les ordres d'enseigne- 
ment, parents d'élèves, étudiants à l'enseignement supérieur, ou représentants du milieu socio-économique. Les vingt-deux membres du Conseil sont nommés par un décret du gouvernement du Québec. Les Commissions Couvrent Chacune un ordre d'enseignement et le secteur de l'éducation des adultes et elles sont Composées de membres nommés par le Conseil suite à une consultation des milieux de l'éducation. Leur mandat est de quatre ans, renouvelable une fois. Ils siègent à titre individuel et non comme porteparole de l'instance professionnelle ou de l'organisme auquel ils appartiennent bien qu'ils doivent en réperCuter les préoccupations et rendre Compte de Ce qui se passe sur le terrain.

Tant la mission du Conseil, que les caractéris tiques des membres qui composent ses instances, obligent à mettre en place une démarche de production des connaissances tout à fait originale. Elle fait appel, d'une part, à une démarche classique de recherche sous la responsabilité des chercheurs rattachés à la permanence du Conseil. D'autre part, les membres des instances de l'organisme, dont les interventions se fondent principalement sur leurs propres pratiques et expériences professionnelles, sont aussi mis à contribution dans la production de l'argumentaire analytique qui sera déployé pour soutenir les recommandations qui seront mises de l'avant dans un avis donné. Par ailleurs, en tant qu'organisme Consultatif auprès $d u$ ministre de l'Éducation et outil de participation démocratique, le Conseil doit aussi solliCiter les opinions du milieu, faire effectuer des recherches et des études et entendre les requêtes et suggestions du public en $m$ atière d'éducation. Pour remplir adéquatement ses objectifs, le Conseil doit pouvoir miser sur sa capacité d'intégration des savoirs savants et des savoirs pratiques. Il doit, en Conséquence, favoriser une interaction dynamique entre les porteurs de Ces deux catégories de savoirs et soutenir une intégration fructueuse des résultats des travaux de recherche et des connaissances provenant des pratiques des acteurs sur le terrain.

C'est dans la mise en œuvre de ce processus Complexe que se trouve la spécificité de son mode de travail qui s'assimile à une démarche de coConstruction des savoirs. Une analyse rigoureuse de Ce mode de travail menée en temps réel et fondée sur une observation systématique des différentes étapes de préparation d'un avis, depuis le choix du thèm e qui sera traité jusqu'à l'adoption de l'avis par les membres du Conseil, n'a malheureusement jam ais fait l'objet d'aucune recherche. C'est pour- quoi, il nous sera impossible de rendre Compte d'une manière fine et rigoureuse des rapports qui se déploient dans ce jeu de face à face entre ces deux ordres de Connaissances portés par deux catégories de producteurs (Chercheurs et praticiens); difficile aussi de démontrer Comment ce jeu d'interactions a mené aux résultats obtenus en terme de Contenus programmatiques et de nouvelles connaissances.

La mission du Conseil exige davantage et ne s'arrête pas ici. En tant qu'organisme de participation démocratique, il se doit d'accomplir son devoir de Consultation très judicieusement. Pour y arriver, il doit prendre les moyens néCessaires pour rappeler au ministère de l'Éducation, aux Citoyens et aux organismes et associations, leurs responsabilités de participer aux Consultations et de répondre aux demandes d'informations et d'enquêtes de terrain qu'il leur adresse. Sous cet angle, cette Cueillette d'information prend une dimension particulière. Tout en enrichissant la dém arche classique de recherche, elle répond ici à d'autres objectifs qui visent à responsabiliser les acteurs de l'éducation à tous les niveaux du système, ainsi que la population en général, à remplir leur devoir de Citoyen. Ce qui se traduit ici par un apport volontaire des membres de la société Civile à la production et à la cirCulation d'informations lorsque sollicités par les recherches et enquêtes du Conseil. La démarche de recherche du Conseil doit aussi se ConCevoir Comme un outil de Consultation démocratique à des fins de Conseil auprès du ministre de l'Éducation et en vue de l'éclairer dans sa prise de décision. On trouve ici un autre aspect de l'originalité de la démarche de recherche du Conseil qui n'est pas présent dans la dém arche classique de recherche. De par Ia Loi qui le fonde, le Conseil doit se donner les moyens de sonder la population et, plus spécifiquement, le monde de l'éducation et de répercuter les opinions ainsi recueillies dans ses avis. Pour le ministre, il s'agit d'un volet important de validation des recommandations que lui soumet le Conseil.

Le pouvoir de Cet organisme consultatif en est un d'influence auprès des décideurs politiques. I s'exerce directement au moment de l'élaboration des politiques éducatives et de l'adoption de nouveaux régimes pédagogiques ou de leur modification, vu l'obligation $d u$ ministre, dans ce dernier cas, de demander l'avis du Conseil. ICi aussi, Ia recherche joue un rôle tout à fait particulier. L'efficaCité de Ce pouvoir repose sur la capacité du Conseil de traduire ses observations et son analyse en orientations pour l'action. Sa crédibilité nécessite de 
pouvoir s'appuyer sur des recherches menées selon les règles de l'art et de les jouxter à une analyse réflexive de ses membres qui soit de haut niveau. L'analyse réflexive que le Conseil préconise s'appuie sur une pensée Critique et pragmatique, sur une vision systémique et prospective, sur l'utilisation de résultats de recherches, sur une connaissance de la Conjoncture nationale et internationale afin de mettre en Contexte les questions traitées. Elle doit mener à la formulation de recommandations opérationnelles axées sur des ajustements ou des changements m ajeurs à apporter pour améliorer le systèm e d'éducation au Québec. Cette forme d'analyse comporte des exigences spécifiques pour les membres qui doivent délaisser la défense de leurs intérêts catégoriels, voire personnels, et être en mesure de traduire leur expérience professionnelle en savoirs d'expérience et de soumettre leurs opinions et leurs perceptions au registre de l'analyse. En même temps, ils doivent faire preuve de capacités d'écoute des préoccupations de leur milieu et pouvoir réperCuter ce qui se passe dans le champ de l'éducation, tout en Contribuant aussi à l'élaboration d'analyses de questions très diverses et parfois éloignées de leurs préoccupations premières. De plus, ils doivent posséder un intérêt pour l'évolution des idées et des Courants de pensée qui animent les débats de société et, en particulier, en éducation.

La mission du Conseil Comporte, par ailleurs, d'autres enjeux de taille. À titre d'exemple, il ne peut se faire le porte-parole direct des groupes particuliers $m$ ais il doit prendre acte de leurs positionnements. II doit être tenu au Courant des grands dossiers de l'actualité en éducation, tout en conservant la distance critique nécessaire au maintien de son autonomie et de saliberté de pensée pour pouvoir jouer son rôle premier d'évaluation de situation, de réflexion Critique, de diagnostic et de recommandation.

Si le Conseil utilise de manière importante les résultats de la recherche académique dans le domaine de l'éducation pour bien documenter les thématiques qu'il traite, il se différencie Cependant des organismes classiques de recherche. En effet, la recherche menée au Conseil vise aussi à soutenir d'une Certaine manière son fonctionnement démoCratique et son rôle d'éclaireur auprès des décideurs politiques en matière d'éducation. De plus, l'expertise de ses chercheurs et la qualité des recherches produites ne sont pas suffisantes à l'exercice de sa mission. Celle-ci repose en grande partie sur les niveaux de compétences des membres des Commissions à pouvoir fonctionner dans un mode de Co-production d'analyses de situation devant ouvrir surdes recomm andations de nature politique, recommandations dont la formulation et l'adoption relèvent d'une autre instance, Celle de la table des membres du Conseil qui Chapeaute tout l'organisme.

Le pouvoir d'influence du Conseil repose entre autre sur sa capacité à faire circuler ses idées tant dans les milieux de l'éducation que dans les autres segments de la société Civile. Une large diffusion publique de ses productions présentées sous différentes formes Constitue un levier pouvant favoriser son influence auprès du Ministre et dans la société Civile. En Ce sens, le Conseil est appelé à jouer, pour ainsi dire, le rôle d'un Centre de liaison et de transfert des Connaissances, un peu à la manière de ceux qui ont été for$m e ́ s$, il y $a$ une quinzaine d'années, $p$ ar le $m$ inistère de I'Enseignement supérieur, de la Science et de la Technologie. Il exerce un rôle de liaison entre la recherche universitaire et les milieux de pratique par le proces. sus même de Co-Construction des savoirs que nous venons de décrire; son rôle de transfert des Connaissances passe par la diffusion de ses productions vers les milieux de pratique et vers les déCideurs politiques et rejoint sa mission d'alimenter et d'animer le débat public dans le champ de l'éducation.

La notion de "passeur"proposée par Jean-Louis Derouet décrit bien, à notre avis, en quoi consiste ce travail de liaison et de transfert. En effet, Cette notion permet de montrer Comment l'interaction, la négociation et la reformulation, sont des activités Constantes du processus de Circulation des savoirs. Selon lui, "Cette approche permettrait de mieux appréhender les rapports complexes entre les formes savantes et les formes ordinaires de la connaissance telles que les acteurs la construisent, mais aussi entre les savoirs académiques et les pratiques pédagogiques et administratives qui posent la question de repérer à la fois Comment la science récupère l'expérience professionnelle et Comment l'activité pratique mobilise les résultats scientifiques" (Derouet, à paraître). Cette fonction de passeur s'applique bien à la mission du Conseil supérieur de l'éducation qui, pour respecter l'esprit qui a présidé à sa Création, se doit de miser sur la Création de liens efficaces entre la connaissance scientifique et la connaissance ordinaire d'une part, et d'autre part, sur une rencontre productive entre les CherCheurs et les acteurs de terrain porteurs de Ces différents savoirs.

L'exercice de Ce rôle requiert des Compétences spécifiques et C'est pourquoi nous en avons fait une dimension de la capacité du Conseil à remplir sa 
mission. Nous y reviendrons. De plus, ce rôle exige un fonctionnement particulier de la part des chercheurs devant travailler dans cette perspective. Ils doivent être en mesure de traduire des savoirs de nature fort différente en provenance des acteurs de terrain et de la recherche scientifique. Ils doivent aussi dégager de ces savoirs ainsi intégrés une analyse socio-politique qui servira de toile de fonds à la formulation de recommandations qui soient Crédibles pour les différentes $C$ aégories d'acteurs Concernés, al premier rang desquels figurent les décideurs politiques. Dans ces deux moments de Circulation des savoirs, tant les Chercheurs que les acteurs du système d'éduc aion sont appelés à exerCer des compétences spécifiques en matière de transfert des Connaissances.

Dans Cette première partie, nous avons voulu faire ressortir l'originalité du mode de travail du Conseil supérieur de l'éducation et la place qu'y occupe la recherche dans la production des idées et des connaissances. Cette originalité repose en bonne partie sur sa démarche de Co-Construction des savoirs où sont mobilisées des Compétences en $m$ atière de production, de traduction et de transfert des savoirs; des Compétences que doivent posséder les chercheurs, mais aussi les acteurs du milieu de l'éducation. La qualité et la crédibilité des travaux d'organismes aviseurs tels que le Conseil, et le niveau de leur impact auprès des responsables politiques et de la société en général, en dépendent. Ces compétences sont davantage requises de la part de personnes appelées à siéger dans des instances chargées d'influencer les politiques publiques. Ils deviennent, pour ainsi dire, des partenaires des Chercheurs tout en demeurant les acteurs du système qu'ils appellent à se transformer suite aux recomm andations qui émergeront de leur travail analytique. Compte tenu du rôle attendu de Ces personnes, nous avons observé que tant Celles qui sont proposées par le milieu de l'éducation que celles qui sont nommées par le ministre pour siéger à la table du Conseil, sont, dans la grande majorité des cas, des personnes qui avaient déjà à leur crédit l'exercice de responsabilités de direction ou de leadership dans le milieu de l'éducation, ou encore qui avaient manifesté un intérêt Certain pour la chose publique.

Comme nous l'avons mentionné d'entrée de jeu, aucune recherche rigoureuse n'a été réalisée jusqu'à Ce jour sur le mode de production des avis du Conseil à partir $d u$ point de vue des Chercheurs et des membres de ses instances. Il faut donc souhaiter que des projets de recherche soient réalisés pour mieux documenter cette démarche. Une démarche tout à fait particulière de production de savoirs en éducation parce qu'elle fait appel à une interaction Continue entre les porteurs de savoirs pratiques et les Chercheurs engagés dans une démarche scientifique.

Nous tenterons maintenant, à l'aide de quelques exemples, d'expliciter plus concrètement le travail propre du Conseil dans la Construction de son discours. Son impact sur l'élaboration des politiques dans le champ de l'éducation fera aussi l'objet de notre examen.

\section{LA PRODUCTION DU DISCOURS DU CONSEIL SUPÉRIEUR DE L.ÉDUCATION ET SON IMPACT SUR L'ÉLABORATION DES POLITIQUES PUBLIQUES}

Pour bien saisir les particularités de la production du discours du Conseil, il faut tenter de Cerner Comment le travail s'organise entre les représentants des milieux de pratique et les chercheurs d'une part, et d'autre part, Comment se Combinent les savoirs pratiques et les savoirs savants dans l'élaboration d'analyses et de propositions visant à éclairer et à orienter l'action des décideurs publics et des milieux de l'éducation.

Nos observations reposent sur notre propre expérience au sein de cet organisme en tant que présidente (1) durant près de six ans (1997-2002). Nous avons à ce titre dirigé l'organisme et supervisé l'ensemble des activités. Nous avons agi Comme porte-parole auprès du milieu de l'éducation et nous avons été redevable au ministre de l'Éducation de la réalisation de la mission du Conseil.

La démarche de préparation d'un avis comporte plusieurs étapes que nous décrirons brièvement afin d'identifier dans la mesure du possible le rôle de chacun des groupes d'acteurs qui y participent. La première étape Consiste à décider des thèmes sur lesquels porteront les avis qui devront être produits dans une année donnée. Le choix de Ces thèmes représente une étape critique. Il repose sur une analyse de la conjoncture à moyen terme en éducation, au Québec et dans le monde, et sur la prise en Compte des dossiers qui sont sur la table du ministre au Cours de l'année, des questions particulières qui font problème et qui demandent des solutions à Court terme ou encore des nouvelles politiques en préparation. La réflexion qui mènera au choix $d u$ thème doit Combiner une vision prospective et un souci d'éclairer le ministre sur des questions qui figurent dans l'actualité de l'éducation au Québec. 
À Cette première étape du processus, nous avons observé que le partage du travail entre les chercheurs et les membres des instances s'effectue la plupart $d u$ temps, de la manière suivante. Les Chercheurs contribuent davantage à l'analyse prospective et à la Cueillette de données de recherche et à l'évaluation de la pertinence de choisir un thème donné au regard de la conjoncture qui prévaut en éduc aion au Québec. De leur Côté, les membres des instances apportent davantage de Considérations témoignant des préoc opations de leurs groupes professionnels respectifs et de leurs perCeptions des problèmes présents dans le milieu de l'éduc aion. Données de recherche et perceptions des acteurs deviennent les matériaux sur lesquels s'appuient les Commissions pour choisir le thèm e qui feral'objet de l'avis. Les chercheurs agissent ici en soutien aux membres des Commissions. C'est finalement la table du Conseil qui a le pouvoir de trancher et de faire le Choix final du thème qui fera l'objet de l'avis dont la préparation revient ensuite à l'une ou l'autre des Commissions selon que le thème Choisi Concerne l'enseignement primaire, secondaire, Collégial, universitaire ou l'éducation des adultes.

En fin de parCours, l'étape finale qui consiste en l'adoption de l'avis préparé par la Commission est du ressort du Conseil. Celui-Ci a tout le pouvoir d'y apporter des modifications et d'ajouter ou de retranCher des recomm andations. La table du Conseil doit dans la mesure du possible fonder ses déCisions sur une vision systémique de l'évolution $d u$ système d'éducation et démontrer un Certain flair politique pour évaluer la pertinence du Contenu tant du point de vue du ministre, que de Celui des acteurs du système d'éducation. Il en va de sa crédibilité publique et de sa légitim ité politique.

Tout en suivant les règles propres à toute dém arche scientifique, les Chercheurs du Conseil doivent tenir Compte dans leur façon de Conduire la recherche et de rassembler les matériaux de l'importance de présenter les résultats de leurs recherches dans une forme qui soit appropriée aux finalités d'un avis qui doit Comprendre des recommandations pour l'action. L'analyse du processus qui conduit à la production d'un avis montre clairement que les problématiques retenues et les Concepts utilisés sont Ceux qui auront été proposés par les chercheurs. Les membres des instances demeurent en retrait de Cette partie du travail et adhèrent, sans en débattre longuement, aux cadres d'analyses proposés par les chercheurs. Il s'est établi une relation de Confiance envers les chercheurs de la part des membres des Commissions et du Conseil. Il arrive rarement que leurs Compétences soient remises en question et, lorsque c'est le cas, il est attendu que la direction administrative $d u$ Conseil fasse en sorte qu'ils soient retirés du dossier. Au Conseil, selon nos observations, les CherCheurs ont un pouvoir d'influence important sur le Choix du cadre analytique qui soutiendra l'argumentaire d'où émergeront les recomm andations de l'avis en préparation.

Concrètement, le processus se déroule à peu près comme suit. Un premier exemple nous servira à le décrire. Il y a quelques années, la question du décrochage scolaire des garçons et de l'écart de réussite entre les filles et les garçons a fait l'objet de vives préoccupations au sein $\mathrm{du}$ monde scolaire et, plus largement de la société québécoise. La ministre de l'Éducation de l'époque a demandé au Conseil de préparer un avis sur Cette question en Centrant l'étude sur les problèmes rencontrés par les garÇons. Les membres du Conseil ont plutôt opté pour une comparaison des facteurs de réussite des filles et de Celle des garçons, indiquant, de ce fait, un positionnement politique à l'effet que les problèmes rencontrés par les garçons ne devraient pas Conduire à négliger la situation des filles. Une fois fixé par le Conseil le choix des orientations à donner au traitement de Ce thème, Ce sont les Chercheurs qui ont eu la responsabilité de colliger les résultats de recherche sur Cette question et de trier les éléments nécessaires à la construction de la problématique qui allait encadrer la formulation du problème ainsi que les dimensions à prendre en compte dans l'analyse. Les chercheurs ont soumis aux membres des Commissions de l'enseignement primaire et de l'enseignement secondaire les résultats de leurs propres recherches et des recherches Consultées en mettant en évidence les dimensions explicatives les plus intéressantes. Ceux-Ci en ont débattu pour bien en Comprendre le sens et la portée et enfin adopter une grille d'analyse.

Cette étape étant franchie, le travail des membres de Ces deux Commissions a porté plus spécifiquement sur l'élaboration des mesures à prendre pour Corriger la situation et soutenir l'amélioration de la réussite des filles et des garÇons. Comme C'est le cas dans la préparation de la grande majorité des avis, l'organisation du travail qui y préside repose sur une séparation claire des fonctions et des expertises entre la production des savoirs savants et Celle des savoirs pratiques. Il est clair, Compte tenu des Composantes de la mission du Conseil que nous 
avons exposées dans la première partie de notre présentation, que Celui-Ci doit s'appuyer sur Ces deux types de savoirs dans l'élaboration des Contenus des recommandations. Dans le $\mathbf{C}$ a de Cet avis, les savoirs pratiques portés par les membres des Commissions ont été davantage mis à contribution dans l'élaboration du plan d'action et dans la désignation des acteurs du système scolaire appelés à les réaliser.

Cependant, un retour sur les étapes de la préparation de Cet avis montre clairement que ce sont les résultats de la recherche qui ont orienté de manière forte le diagnostic Concernant les problèmes identifiés et servi d'outils pour valider le choix des positionnements des membres des Commissions et du Conseil. Les nombreux débats qui ont suivi la parution de cet avis (Québec, 1999) ont montré l'importance de s'appuyer sur les résultats de recherche pour légitimer Certaines des recom mandations auprès du monde scolaire et de la population en général. Ainsi, la mixité des classes et l'absence d'hommes dans le corps enseignant au primaire ont souvent été évoquées comme causes des problèmes scolaires des garçons, lors de débats publics. La présentation de résultats de recherches menées sur Ces questions au Québec et dans d'autres pays, a permis de démontrer et de Convaincre, du moins en bonne partie, qu'il ne s'agissait pas de facteurs déterminants et de réorienter les prises de position sur d'autres facteurs explicatifs plus significatifs. La recherche dans Ce cas-Ci a permis de déConstruire des perCeptions premières très fortes et de réorienter l'action sur d'autres causes (par exemple, les mesures spécifiques en lecture et écriture ; le décodage des stéréotypes sexuels dans la conception des rôles scolaires et le rôle des pères dans l'éducation de leurs fils, etc.).

Les analyses et les reCommandations du Conseil sur cette question ont franchi le terrain du politique et servi de références centrales au ministre dans la définition des propositions qu'il $a \mathrm{~m}$ is de l'avant pour remédier aux problèmes identifiés. En effet, plusieurs mesures et programmes spéciaux (le programme "Agir autrement"; l'élaboration de plans de réussite pour chaque établissement) ont été mis en place par le ministre de l'Éducation pour donner suite aux recommandations du Conseil. Nous avons iCi un exemple probant des liens étroits qui ont existé entre la démarche de recherche, la définition des pistes d'action par les membres des instances du Conseil représentant les milieux de pratique et l'élaboration de mesures et de politiques au sein $d u$ ministère de l'Éducation. Cette question a occupé la place publique à la suite de la publication de cet avis et elle Constitue encore aujourd'hui une préoccupation qui rejoint l'ensemble de la société québécoise.

Dans le cas de figure que nous venons d'évoquer, C'est un constat de situation venant du terrain sur le taux de décrochage scolaire des garçons qui a Conduit les déCideurs politiques à demander au Conseil supérieur de l'éducation de lui donner un avis sur Cette question. Un autre cas de figure que nous évoquerons maintenant, fait référence au mandat d'initiative du Conseil. Celui-Ci peut, à partir de sa propre évaluation de la conjoncture qui prévaut en éducation, choisir de préparer un avis au ministre sur une question qu'il juge nécessaire de prioriser dans l'agenda politique. Dans ce cas de figure, Ia thématique est Choisie par le Conseil et l'argumentaire vise à convaincre le ministre de faire les suivis nécessaires soit $p$ ar le biais de modifications aux règlements pédagogiques ou encore au niveau légis latif, ou par l'implantation de toute autre mesure relevant de sajuridiction. L'exemple que nous avons Choisi de présenter pour illustrer le mandat d'initiative du Conseil fait référence au Rapport sur l'état et les besoins de l'éducation qu'il a déposé au Ministre en 1998, à l'effet d'introduire une nouvelle dimension dans les finalités de la formation. Dans ce rapport il était proposé d'inscrire l'éducation à la Citoyenneté Comme Composante des nouveaux programmes de formation au primaire et au secondaire et comme finalité de formation.

Cet exemple nous permettra de mettre en évidence une autre forme d'apport de la recherche. Elle Consiste, dans ce cas, à explorer de nouveaux Champs d'intervention en éducation et à proposer un nouveau corpus de références en $m$ atière de finalités de formation. L'examen des retombées de ce rapport (Québec, 1998a) à la suite de sa publication et de sa diffusion publique montre que la problématique et les concepts proposés par le Conseil, ont été repris en grande partie par les divers Comités Chargés d'élaborer les programmes au ministère de l'Éducation ainsi que par les intervenants de la société Civile qui ont appuyé la recommandation du Conseil d'inscrire l'éducation à la Citoyenneté dans les finalités de formation.

En effet, il y a quelques années, au moment de la parution de ce rapport, le champ de l'éducation à la Citoyenneté était tout à fait nouveau, du moins au Québec, et fort différent dans sa perspective de l'éducation civique figurant dans les programmes 
auparavant. Cette nouvelle problématique allait néCessiter d'inscrire de nouveaux objectifs à la fois dans les finalités globales et dans les contenus des programmes du primaire et du secondaire. Les influences qui ont présidé à ce travail sont venues de mouvements similaires observés au sein de la Communauté européenne et de pratiques déjà en $œ u v r e d a n s$ Certains milieux de l'éducation tant dans Ces pays qu'au Québec, mettant de l'avant la valorisation d'une formation à la Citoyenneté visant à moyen terme à redonner à la société Civile les outils nécessaires à l'exercice de la démocratie.

Tout le mouvement qui a initié Ce Changement provient en grande partie de débats d'idées initiés par le Conseil. Si le Conseil, à l'aide de son équipe de recherche, a construit son argumentation à partir de recherches menées dans plusieurs pays, il s'est Cependant appuyé sur une Conjoncture spéCifique au Québec pour justifier cette recomm andation. Le prinCipal élément de Cette justification est venu de la Commission des États généraux sur l'éducation (1995-1996) qui venait de déposer son rapport au ministre de l'Éducation. La Commission y recom$m$ and ait d'inscrire la mission de socialisation Comme finalités de l'éducation, avec celles d'instruire et de qualifier. La socialisation y était définie sous l'angle de "la transmission des valeurs qui fondent notre société démocratique (l'égalité, les libertés fondamentales, la justice, la coopération et la solidarité) et le respect des institutions Communes. Elle prépare les individus à l'exercice de la Citoyenneté en leur apprenant leurs droits et devoirs, le respect des règles communes et l'ouverture à la diversité." (Québec, 1996) Ces objectifs furent explicités et Concrétisés par le Conseil dans son rapport Éduquer à la citoyenneté (Québec, 1998a). À peine quelques années plus tard, les objectifs proposés ont été insCrits dans les domaines d'apprentissage et les domaines généraux de formation proposés dans la réforme de l'école québécoise.

Enfin, il est important de mentionner que la dernière décennie a été $m$ arquée dans le champ de l'éducation par des changements majeurs inspirés de la recherche en éducation et de l'intervention de la société Civile, Celle-Ci s'exprimant tant par la voix de la Commission des états généraux sur l'éducation que par celle du Conseil supérieur de l'éducation. Notons aussi qu'environ $85 \%$ des 2000 mémoires soumis à Cette Commission, lors de ses audiences à travers le Québec, ont Cité au moins une référence provenant des avis du Conseil. Le Plan d'action $\mathrm{m}$ inistériel pour la réforme de l'éducation, Prendre le virage du succès (Québec, 1997), a repris sous la forme de sept lignes d'action, l'essentiel des dix Chantiers prioritaires proposés par la Commission qui s'était, à son tour, fortement inspirée des travaux du Conseil supérieur de l'éducation (Québec, 1995b). En 1998, le gouvernement du Québec adoptait la Loi sur l'instruction publique (loi 180) qui allait Concrétiser les grandes orientations de ce Plan d'action et donner le Coup d'envoi de la présente réforme de l'éducation.

Il est difficile d'établir clairement la part d'influence de chacun de ces acteurs dans l'élaboration et la $m$ ise en application des réformes ou des Changements en éducation, aucune étude rigoureuse et systématique n'ayant été réalisée encore sous cet angle. Mais il m'apparaît clair, qu'en plus des acteurs, le ministère de l'Éducation en tant qu'appareil d'État a joué le rôle de grand arbitre dans les choix qui ont été faits par la suite à partir des recommandations de la Commission des états généraux sur l'éducation et du Conseil supérieur de l'éducation. En effet, durant la décennie (1995-2005), sept $m$ inistres de l'Éducation se sont succédés provenant de deux gouvernements différents, soit le Parti québécois, de 1995 à 2003 et par la suite, le Parti libéral. Cependant, tous Ces ministres ont maintenu le cap sur le cheminement de Cette réforme et n'ont pas modifié les grandes orientations, du moins jusqu'à ce jour. Ils ont Confié sa mise en application aux établissements et la rédaction des programmes au sous-ministre associé à l'enseignement primaire et secondaire et à des comités Composés de fonctionnaires $d u$ ministère et de professeurs des Facultés des sciences de l'éducation.

La situation québécoise suggère le commentaire suivant. La plupart des études qui ont porté sur le rôle de l'État en éducation se sont intéressées davantage à l'analyse de contenu des politiques et aux conséquences de leur mise en application sur le système d'éducation. Il faudrait encourager la réalisation de recherches portant sur les processus de prise de décision dans l'élaboration des politiques et des divers programmes en éducation et sur le rôle de chacun des acteurs. Parmi Ces acteurs, il serait opportun de porter une attention particulière au rôle de la bureaucratie d'État dont relèvent les comités $m$ inistériels chargés d'élaborer les politiques et les programmes qui les accompagnent. Nous Constatons que peu d'études ont été menées sur la nature des pouvoirs de l'ensemble des acteurs qui œuvrent dans le champ de l'éducation et sur la façon dont Ces pouvoirs se partagent entre la société Civile et le gouvernement.

80 Revue française de pédagogie, n॰154, janvier-février-mars 2006 


\section{PISTES POUR UNE CONSOLIDATION DES RAPPORTS ENTRE LA RECHERCHE, LES MILIEUX DE PRATIQUE ET LES POLITIQUES ÉDUCATIV ES}

Notre analyse du mode de travail du Conseil supérieur de l'éducation et l'expérience qui a été la nôtre à travers diverses commissions gouvernementales en éducation (2) au Cours de la dernière décennie, nous suggèrent Certaines pistes pour Consolider les rapports entre la recherche, les milieux de pratique et les acteurs politiques chargés d'élaborer les politiques éducatives. La priorité, selon nous, devrait être accordée au renforCement des capacités professionnelles des acteurs qui interviennent en première ligne dans le champ de l'éducation dans le but de mieux les outiller afin qu'ils puissent Contribuer davantage à la Construction des savoirs en éducation. Le Conseil, dans l'un de ses avis, a recommandé que l'une des Composantes du référentiel (Québec, 2000) de formation des enseignants du Collégial (post-secondaire) vise l'acquisition de compétences qui préparent l'enseignant à la maîtrise de l'évolution de sa pratique et à sa capacité de pouvoir contribuer au devenir de la profession enseignante, soit à Court, moyen, ou long terme. Ces Compétences visent l'acquisition des outils nécessaires pour permettre à l'enseignant d'analyser ses pratiques et de participer au développement de Connaissances tant pédagogiques, que disciplinaires et technologiques.

En tant qu'acte réflexif, la pratique de l'enseignement requiert une démarche de réflexion dans l'action et sur l'action. On dira que l'enseignement se professionnalise dans la mesure où il prend de plus en plus ses distances par rapport à l'application mécanique de techniques; dans la mesure aussi où il intègre davantage une activité d'élaboration de stratégies qui s'appuient sur des savoirs Constitués et, selon Marguerite Altet, sur le développement "d'une expertise de l'action en situation professionnelle »(Altet, 1996, p. 29). Philippe Perrenoud (1996, p. 206) nommera Cette démarche de réflexion Comme étant une démarche de lucidité profession. nelle qui requiert de développer un habitus profes. sionnel autour de l'analyse des pratiques en vue de les modifier au besoin. Pour y arriver, il est essentiel de se mettre en situation de prise de Conscience de son propre mode de fonctionnement. Tant le "savoir analyser " que l'habitus professionnel font partie de Ce que Michel Develay nomme "la Conscience des ressorts de son action".

Par ailleurs, la m aîtrise de l'évolution de la pratique enseignante et la contribution au devenir de la pro- fession enseignante requièrent aussi de pouvoir Contribuer au développement des Connaissances d'une manière qui aille au-delà de l'auto-analyse des pratiques et d'une démarche de réflexion. S'il est souhaitable que tous les enseignants prennent Connaissance de la recherche et de ses résultats pour les réinvestir dans leur pratique, ils devraient aussi être $m$ is à contribution d'une façon plus active en vue de fournir un apport davantage significatif à la théorisation des savoirs d'expérience pédagogique, c'est-à-dire à la transposition des savoirs de sens Commun en savoirs savants.

Ces propositions nous apparaissent fort pertinentes non seulement du point de vue des objectifs de la formation des enseignants et du développement de leur identité professionnelle, comme Cela a été proposé dans les divers documents publiés sur Ces questions. Elles comportent en effet un intérêt supplémentaire et non négligeable, Compte tenu de l'analyse que nous venons de proposer sur les rap. ports entre les milieux de pratique et l'élaboration des politiques en éducation, à partir de la description $d u$ mode de travail du Conseil. L'acquisition de Ce type de Compétences et de Connaissances permettrait aux enseignants de tous les ordres d'enseignement de disposer des outils nécessaires pour intervenir sur les orientations des politiques en éducation avec la distance critique nécessaire; elles favoriseraient aussi l'acquisition des Connaissances nécessaires pour pouvoir non seulement influencer $m$ ais aussi proposer des alternatives innovatrices allant au-delà de la perspective défensive et Corporatiste, dans laquelle ils se situent trop souvent. La maitrise de telles compétences devraient aussi permettre aux acteurs des divers milieux de l'éducation appelés à siéger sur des instance Consultatives du gouvernement, Comme le Conseil supérieur de l'éducation ou tout autre comité ministériel, moins dépendants des spécialistes et des experts, et mieux en mesure de pouvoir débattre des résultats de recherche et des propositions d'analyse en toute Connaissance de cause.

À Ce titre, les Facultés de sciences de l'éducation ont un rôle primordial à jouer dans la formation initiale et le perfectionnement des personnels scolaires. En Conséquence, il serait souhaitable qu'elles poursuivent et Consolident leur association avec des écoles primaires et secondaires; qu'elles introduisent dans leurs programmes de formation, une approche pédagogique favorisant une meilleure intégration des savoirs pratiques et des savoirs savants et qu'elles encouragent l'innovation et 
l'expérimentation de nouveaux modèles de gestion et de formation ainsi que leur évaluation. Les programmes universitaires de formation des maîtres devraient ac order une place spécifique à la formation à la recherche dans les programmes de premier Cycle (Québec,1993 \& 1998b) pour inciter les enseignants à prendre connaissance des résultats de la recherche et à les réinvestir dans leurs pratiques, à développer la c a acité d'analyse Critique de leurs propres pratiques et des pratiques éduc aves en général, et à contribuer à leur renouvellement.

Dans la foulée des nouvelles politiques en éduc aon et Considérant les changements majeurs qui traversent son mode de gestion et d'organisation, de même que ses programmes et ses méthodes pédagogiques, le système scolaire se situe au Québec àıne Croisée des chemins. Les universités ont ici un rôle Central à y jouer, Craelles portent une double responsabilité : Celle de la formation des maitres et des directions d'établissements scolaires et Celle de la recherche en éduc aon. C'est une lourde responsabilité dont la prise en charge requiert de l'institution universitaire qu'elle se Conçoive Comme un lieu stratégique de Croisement de Ces divers types de savoirs et de production de nouvelles connaissances nécessaires à l'élaboration de politiques éducatives et à leur ajustement.

Cette perspective sur le rôle des universités ouvre sur une seconde piste d'action susceptible de favoriser la Consolidation des rapports entre la recherche et les pratiques enseignantes. Elle oblige à examiner le statut des sciences de l'éducation et leur place dans l'institution universitaire. En effet, le développement de la recherche en éducation et la reconnaissance de la légitimité du champ de l'éducation en tant que domaine de Connaissance et champ du savoir doivent être mieux soutenus. Au Québec, la situation à cet égard s'est beaucoup améliorée depuis quelques années mais elle demeure encore fragile. Les débats qui ont eu lieu dans nos universités au moment des modifications des programmes de formation des enseignants au primaire et au secondaire ont été vifs entre les facultés disciplinaires et les facultés des sciences de l'éducation. Bien que la situation aille en s'améliorant, ces facultés (anciennement des éColes normales intégrées aux universités dans les années 1969-1970) demeurent encore aujourd'hui les mal-aimées des universitaires et des universités et elles sont trop souvent marginalisées dans le champ proprement académique.

Le développement récent de plusieurs Centres de recherche et la Croissance du financement de la recherche en éducation témoignent de Ce Changement important. Le besoin de connaissances en éducation est, par ailleurs, très ressenti et nous observons encore de graves lacunes au Québec. Récemment, l'on a assisté à une multiplication des projets de recherche sur le développement du CurriCulum, la réussite éducative, les modes de gestion scolaire, les politiques en éducation, le système d'éducation et son organisation, les pratiques d'enseignement, etc. Pour mieux soutenir ce développement et les particularités propres aux démarches de recherche en éducation, le Conseil a soutenu l'idée de la valorisation de nouveaux modèles de recherche qui répondraient mieux aux besoins propres au champ de l'éducation. Il a aussi souhaité que soit renforcé le support des organismes subventionnaires à ces types de recherche. Nous pensons ici à la recherche-action, à la recherche Collaborative, à la recherche en partenariat universités. écoles dans l'esprit du programme de subventions Connu sous le nom d'Alliances de recherche universités-Communautés (ARUC). Ce programme accorde un soutien financier à une série d'activités de recherche, de formation et de Communication novatrices «qui accentueront l'apprentissage mutuel et la Collaboration horizontale entre les organismes de la Communauté et les universités ; qui Contribueront au développement social, Culturel et/ou économique des Communautés; qui enrichiront la recherche, les méthodes d'enseignement et les programmes dans les universités et renforCeront la capacité des Collectivités de prendre les déCisions et de résoudre les problèmes". À notre avis, il s'agit ici d'une voie prometteuse pour le développement de la recherche en sciences de l'éducation en association avec les milieux de pratique, susceptible aussi de maximiser l'impact social de la recherche et d'assurer un meilleur soutien à l'évolution continue du système d'éducation et à l'élaboration des politiques éducatives. Rappelons que la "Politique québécoise de la science et de l'innovation" (Québec, 2001C) a fait une place à l'innovation sociale comme Créneau de recherche à développer et l'innovation pédagogique fait partie des types d'innovation à soutenir.

Par ailleurs, la société québécoise dans son ensemble doit pouvoir Comptersur la recherChe pour évaluer et améliorer son système d'éducation et mettre en place les politiques nécessaires pour Ce faire. C'est pourquoi, dans son rapport annuel 2000 2001 au ministre de l'Éducation, le Conseil a proposé "un axe d'intervention qui serait susceptible de soutenir l'État dans sa fonction de gouverne de l'ensemble du système d'éducation [...] Le Conseil

82 Revue française de pédagogie, n¹54, janvier-février-mars 2006 
Croit nécessaire de mettre en place un dispositif Concerté de veille et de diffusion de l'innovation en éduc aion. "(Québec ,2001a,p. 93)

\section{EN GUISE DE CONCLUSION}

II nous apparaît opportun en terminant de rappeler le souhait impératif formulé par le Conseil dans son rapport annuel 1994-1995 (Québec, 1995a) parce qu'il renforce l'idée de l'université Comme lieu stratégique de Création et de renforCement des rapports entre la recherche, les pratiques et les politiques en éducation.

" Il paraît au Conseil d'une importance capitale que les universités privilégient dans leur rôle de développement social, Celui de fournir tout l'éclairage qu'elles sont capables d'apporter en éducation, quand et où il le faut, à la fois dans des équipes de recherche-action sur le terrain, dans les grands débats publics et dans l'analyse indépendante des politiques d'éducation." (Québec, 1995a,p. 80)

Le Conseil a aussi réfléchi à la façon dont il pourrait mieux Contribuer à soutenir les acteurs du système d'éducation à tous les échelons, depuis le ministre de l'Éducation, dont il relève en premier lieu, jusqu'aux acteurs de première ligne. Dans son premier plan stratégique (Québec, 2001 b) préparé à la demande du gouvernement, il a été appelé à proposer des indicateurs devant permettre d'évaluer com ment il remplit sa mission et répond aux mandats qui lui sont Confiés. Il est intéressant de noter la place importante qu'y occupe la recherche comme indicateur de la qualité de son travail: "la production de rapports de recherche qui fondent la réflexion et présentent des synthèses de Connaissances sur un sujet en mettant l'accent sur l'aspect novateur et sur les modèles porteurs d'avenir ici et ailleurs dans le monde; les réalisations de Consultations sous forme d'enquêtes, d'entrevues, d'audiences, d'appels de mémoires... qui assurent l'ancrage de la production du Conseil et offrent des balises quant à la pertinence des orientations proposées et à l'opportunité des pistes d'action. " (Québec, 2001 b, p. 21)

Il a proposé d'autres indicateurs pour mesurer son influence en terme de portée effective de ses avis Compte tenu de son rôle de Conseiller d'État. Ceux-Ci renvoient notamment à la mesure de l'influence du Conseil dans "l'avènement d'une politique ou l'ajus tement d'une politique en réponse à une recommandation du Conseil. "(Québec, 2001 b, p. 22) Tout en reconnaissant que la déCision en $m$ atière de politiques publiques relève de plusieurs facteurs, il n'en demeure pas moins qu'il serait souhaitable qu'une analyse de l'influence du Conseil soit faite. De telles études d'impact permettraient d'enrichir la compréhension des rapports qui se jouent entre la recherche, les milieux de pratique et l'élaboration de politiques publiques. Le Conseil supérieur de l'éducation se révèle être un Iaboratoire exceptionnel à ce titre.

Céline Saint-Pierre saint-pierre.celine@nternet.uqam.ca Université du Québec à Montréal

\section{NOTES}

(1) Ce poste Correspond à Celui de président-directeur général et requiert une implication à temps plein. Le président ou la prési dente du Conseil est nommée suite à un décret du Conseil des $m$ inistres du gouvernement du Québec.
(2) Présidente du Conseil supérieur de l'éducation (1997-2002), j'ai été $m$ embre de la Commission des états généraux sur l'éducation (1995-1996) et plus réCemment, membre du Com ité d'experts sur le financement de la formation Continue (2003-2004).

\section{BIBLIOGRAPHIE}

ALtet M. (1996). "Les Compétences de l'enseignant professionnel: entre savoirs, schèmes d'action et ad aptation, le savoir analyser ". In L. Paquay, M. Altet, É. Charlier \& P. Perrenoud, Former des enseignants professionnels: quelles stratégies? Quelles compétences? Bruxelles: De Boeck, p. 27-40

DeRouet J.-L. (à paraître). "De la notion de transfert à la Celle de Circulation des savoirs". In La circulation des savoirs en éducation: entre recherche et pratique. Actes du Colloque de Pointe-à-Pitre, 2001.
Perrenoud P. (1996). "Le travail sur l'habitus dans Ia form ation des enseignants: analyse des pratiques et prise de Conscience». In L. Paquay, Marguerite Altet, Évelyne Charlier \& Philippe Perrenoud, Former des enseignants professionnels: quelles stratégies? Quelles compétences? Bruxelles: De Boeck, p. 181-207.

QuÉBEC : CoNSEIL SUPÉRIEUR DE L'ÉdUCATION (1993). La gestion de l'éducation: nécessité d'un autre modèle. Rapport annuel 1991-1992 sur l'état et les besoins de l'éducation. Québec : CSE. 
QuéBec : Conseil supéRIEUR de L'ÉduCATION (1995a). Vers la $m$ aitrise $d u$ changement en éduc aion. Rapport annuel 1994-1995 sur l'état et les besoins de l'éducation. Sainte-Foy : CSE.

Québec : Conseil supérieur de L'éducation (1995b). Pour la réforme du système éduC aif: dix années de Consulta tion et de réflexion. Avis au ministre de l'Education. Québec : CSE.

QuÉBEC : MINISTÈre de L'ÉduCATION, DES LOISIRS ET DU SPORT (1996). Rénover notre système d'éduc aion : dix chantiers prioritaires. Rapport final de la Commission des États généraux sur l'éducation. Québec : MELS. Document disponible sur Internet à l'adresse: http: //www.mels.gouv.qc.ca/etat-gen/rapfinal/tmat.htm (Consulté le $3 \mathrm{~m}$ ars 2006).

Québec : Ministère de L'Éducation, DES LoISIRS ET DU SPORT (1997). Prendre le virage du suc es: plan d'action ministériel pour la réforme de l'éduc aion. Québec: MELS. Document disponsible sur internet à l'adresse : $h t t p: / / w w w . m$ els.gouv.qC.ca/REFORME/reform e.htm (Consulté le $3 \mathrm{~m}$ ars 2006).

QuÉBEC : CONSEIL SUPÉRIEUR DE L'ÉDUCATION (1998a). Éduquer à la Citoyenneté. Rapport annuel 1997-1998 sur l'état et les besoins de l'éducation. Québec : CSE.

Québec: Conseil SupéRIEUR de L'Éducation (1998b). Recherche, création et form ation à l'université: une articulation à promouvoir à tous les cycles. Avis au ministre de l'Éducation. Sainte-Foy : CSE.
QuéBec : Conseil supérieur de L'éducation (1999). Pour une $m$ eilleure réussite scolaire des garçons et des filles. Avis au m inistre de l'Éducation. Québec : CSE.

QUÉBEC : CONSEIL SUPÉRIEUR DE L'ÉDUCATION (2 0000$)$. La form ation du personnel enseignant au Collégial: projet collec tif enraciné dans le milieu. Avis au ministre de l'Éducation. Sainte-Foy : CSE.

QUÉBEC : ConseIL SUPÉRIEUR DE L'ÉDUCATION (2001a). La gouverne de l'éducation : logique marchande ou processus politique? Rapport annuel 2000-2001 sur l'état et les besoins de l'éducation. Sainte-Foy : CSE

Québec: Conseil SupéRIEUR de L'Éducation (2001b). Pour mieux conseiller le ministre de l'Éducation et l'État: observer, Consulter, approfondir. Plan stratégique 2001-2003. Québec: CSE.

Québec : Ministère de LA Recherche, de la Science et dE LA TeChNologie (2001C). Politique québéco ise de la science et de l'innovation: savoir changer le monde. Québec : MRST. Document disponible sur Internet au format PDF à l'adresse : http://www.mdeie.gouv.qc.ca/mderContent/000021780000/upload/publications/pdf/scienc e teChnologie/fr/pqsi/mrst_savoirChanger.pdf (Consulté le $3 \mathrm{~m}$ ars 2006).

Saint-PierRe C. (2004). "Le Conseil supérieur de l'éducation: le projet initial du rapport Parent et sa mise en $œ u v r e$ depuis 1964 ". Bulletin d'histoire politique, vol. $12, n^{\circ} 2$, p. $95-105$.

Site Internet du Conseil supérieur de l'éducation de Québec : http://www.Cse.gouv.qC.ca (Consulté le 24 mars 2006). 